\title{
Statistical Compact Model Parameter Extraction Strategy for Intrinsic Parameter Fluctuation
}

\author{
B. Cheng, S. Roy, and A. Asenov \\ Dept. of Electronics \& Electrical Engineering, University of Glasgow, U.K. \\ \{b.cheng|s.roy|a.asenov\}@elec.gla.ac.uk
}

\begin{abstract}
A reduction in the number of parameters involved in the statistical compact model parameter extraction is necessary for the development of scalable compact modelling strategy. The impact of size of parameter set on the quality of statistical compact modelling is investigated in detail by HSPICE simulation at both device and circuit levels. Principal component analysis (PCA) is employed on a two- parameter set to demonstrate the possibility to corporate it into scalable statistical compact modelling strategy.
\end{abstract}

\section{Introduction}

Intrinsic parameter fluctuations (IPF) [1], which arise from the discrete nature of matter, have become one of the major sources of statistical device variability in the decananometer regime. The design of robust circuits in the presence of IPF requires statistical circuit simulation and verification. Since compact models acts as a bridge between designer and foundry, reliable strategies to integrate IPF information into compact models are required. Previously, a statistical compact model parameter extraction strategy was developed to transfer the variability information obtained by 3D 'atomistic' simulation into BSIM compact models [2-3]. The purpose was to precisely reproduce atomistic simulation results, and 7 parameters were selected to optimally capture the effects introduced by random dopant fluctuations (RDF) [3]. Device size is a determinant factor in IPF magnitude, but empirical nature of, and, the correlations between some of the chosen 7 parameters, make it very difficult to develop parameter sets scalable over a range of device sizes. As a result, each sized device requires a completely new statistical parameter extraction.

\section{Impacts of Parameter Set on Statistical Compact Model Parameter Extraction}

Successful development of scalable compact model parameter sets calls for a reduction in the number of parameters used (to make the procedure logistically feasible), retaining parameters with physical meaning. Although the statistical results may not then precisely reproduce every device characteristic, they must be accurate enough to reproduce statistical device behaviour. The benefit of such approach will be the ease of integrating size effects into such a parameter set. In this study, previous results based on the extraction of 7-parameter sets act as a benchmark to indicate how 
parameter set choice can affect statistical compact modelling results.

Fig. 1 shows the impact of RDF on the $I_{D^{-}} V_{G}$ characteristics of $35 \mathrm{~nm}$ physical gate length devices. Lack of inversion charge screening results in significant subthreshold current variation, while increased screening at higher gate voltages reduces the magnitude of the on-current variations - effects well modelled in BSIM4 by the 7-parameter set of fig. 2.
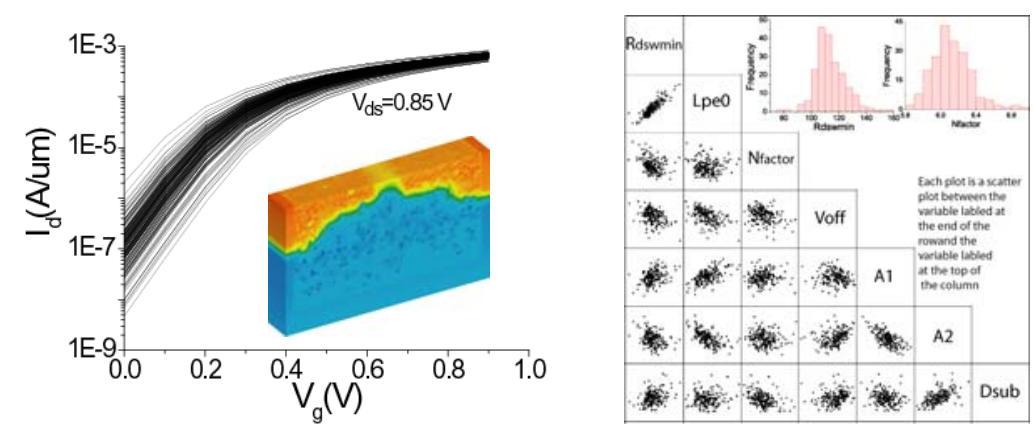

Figure 1: Gate Characteristics of 200 macro- Figure.2: Scatter plots between two scopically identical $35 \mathrm{~nm}$ MOSFETs, mapped parameters and distributions obtained by 'atomistic' simulator. (Inset: of typical parameters, obtained by the potential distribution with the detailed 7 parameter set extraction approach. positions of dopants are considered)

However, the dominant effects of RDF are found to be well modelled simply by $V_{T}$ variation. Figs. 3 show the impact of different sized parameter sets on the quality of the statistical compact modelling. If only $v t h 0$, the basic $V_{T}$ parameter, is used, the mean relative $I_{O N}$ error over an ensemble of statistical fits is $14 \%$. This reduces to $6 \%$ when voff, a subthreshold fitting paramete, is added, and to $4 \%$ when the S/D resistance parameter $r d s w m i n$ is introduced (to account for S/D resistance variation caused by RDF). The previous approach using 7 parameters generates a mean error of $0.6 \%$.

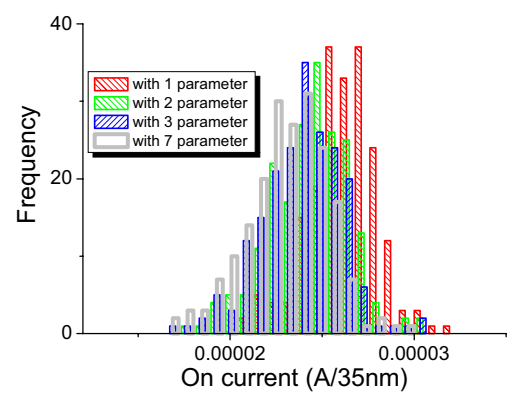

(a)

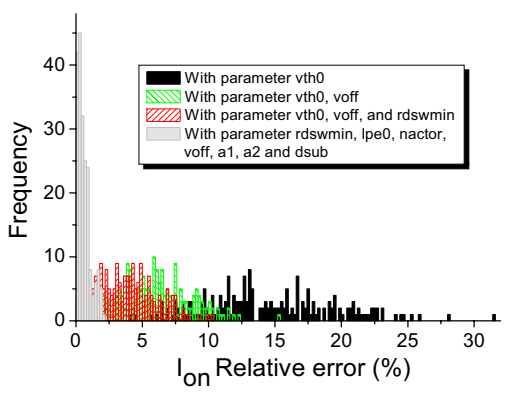

(b)

Figure 3: (a) On current distributions generated by different parameter sets. (b) On current error distribution against different parameter sets 
Fig.4 implies that the physical meaning of key parameters, vtho and voff, is retained during each stage of parameter extraction. The (fig. $4 \mathrm{a}$ ) variance of $v$ th 0 between the 1 and 2-parameter sets is because as a lone parameter $v$ th 0 must account for both $I_{O N}$ and $I_{O F F}$ fluctuations, whilst in the 2-parameter set voff can account for subthreshold current fluctuations.
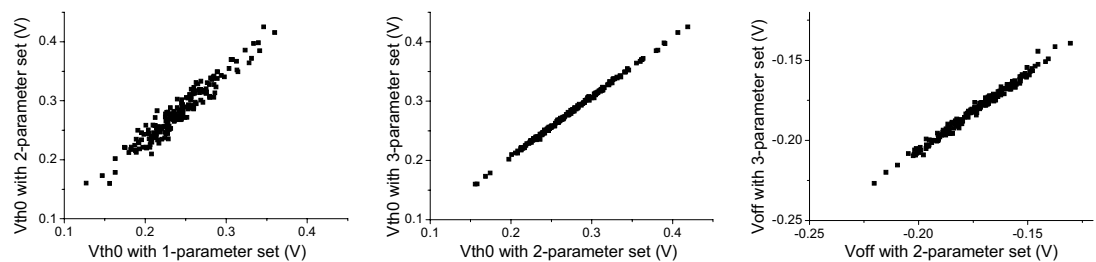

Figure 4: The correlations between the same parameter at different parameter sets

One critical result of transistor IPF is the degradation of SRAM static noise margin (SNM) shown in fig.5a. The impact of the choice of parameter set size on SNM modelling is shown in fig.5b. The relative errors on the calculated SNM mean over an ensemble of devices, $\mu_{S N M}$, (compared to the 7 parameter model) are all within $3 \%$, while the relative errors on $\sigma_{S N M}$ are $20 \%, 7.6 \%$ and $4.8 \%$ for the 1,2 , and 3 -parameter sets respectively.

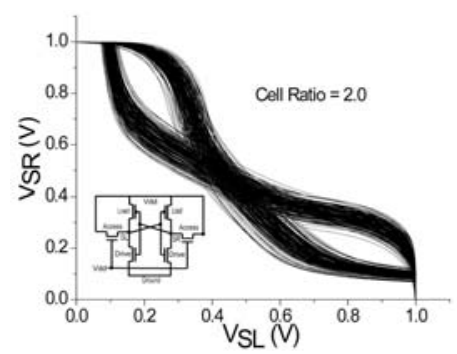

(a)

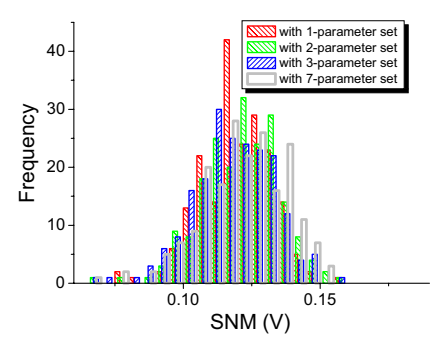

(b)

Figure 5: (a) The static transfer characteristics of 200 statistical SRAM circuit simulations (Inset: Circuit schematics of CMOS SRAM) (b) The distributions of SNM with different parameter sets.

\section{Principal Component Analysis of Parameter Set}

Many of the parameters in device compact model are not statistically independent. In order to guarantee that device used in circuit simulation is 'real', the correlations between compact model parameters should be preserved when Monte Carlo circuit simulation is carried out. Previously, this was achieved by employing the statistical compact model library [3] which was created directly from the statistical compact model parameter extraction. If the correlations between statistical parameters are reasonably similar over a range of device sizes. PCA can be applied to generate scaled statistical compact model parameters. As a first step, PCA is employed on the 2parameter set. The comparison between extracted parameter and random generated 
parameter through PCA is shown in fig.6, the errors in $\mu$ are less than $1.5 \%$ for both $v$ th 0 and voff, where the errors on $\sigma$ are around $6 \%$.

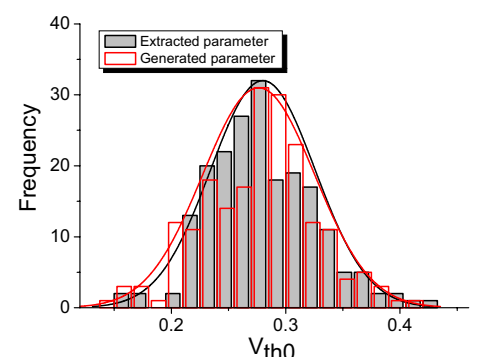

(a)

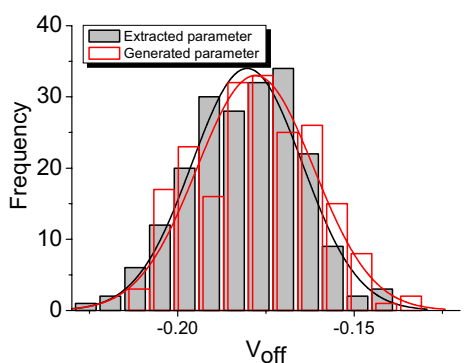

(b)

Figure 6: The distribution of (a) vth0, (b) voff over ensemble of 200 devices,

Fig.7 demonstrates the correlation between vth0 and voff has been well preserved by PCA. For this particular case, the mean relative $I_{O N}$ error over an ensemble of statistical fits is less than $1 \%$, and the error on $\sigma$ is around $8 \%$, with PCA approach generates more variation.

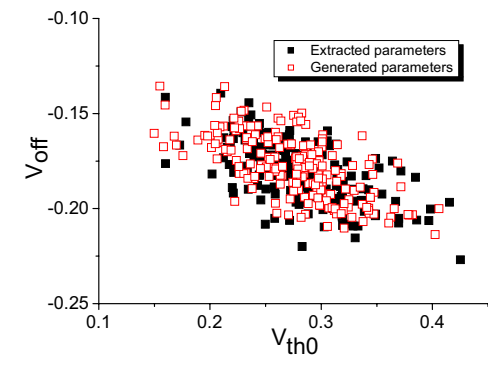

(a)

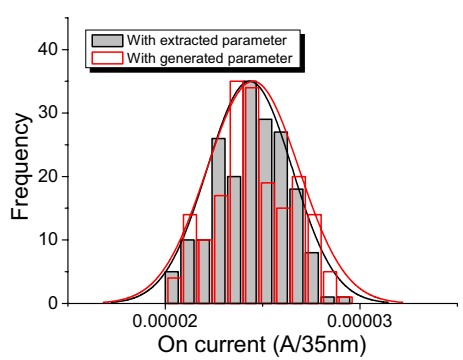

(b)

Figure 7: (a) The scatter plot between vth0 and voff. (b) The on current distributions

\section{Conclusions}

It is crucial to integrate IPF into compact models in order to design IPF aware circuits in the decananometer regime. We have demonstrated that it is possible to select a limited number of BSIM parameters, each with direct physical meaning, to adequately describe the effects of RDF in a real circuit, and PCA can be applied to decouple the correlation between above parameters. These are good starting points for more general integration of IPF size effects into scalable statistical compact models.

\section{References}

[1] A. Asenov, et al., IEEE Trans Electron Dev 501837 (2003)

[2] BSIM4 Manual. From http://www-device.eecs.berkeley.edu

[3] B. Cheng, S.Roy, et al., Solid State Elec., 49, 740 (2005) 